\title{
Application of multilevel analysis approach in management theory
}

\author{
S. Morteza Ghayour ${ }^{a^{*}}$, Shamsodin Nazemi ${ }^{b}$, Fariborz Rahimnia $^{c}$ and Mohammad Lagzian $^{\mathrm{c}}$
}

${ }^{a}$ PhD Student of Organizational Behavior, Faculty of Economic and Administrative Science, Ferdowsi University of Mashhad, Mashhad, Iran ${ }^{b}$ Professor, Faculty of Economic and Administrative Science, Ferdowsi University of Mashhad, Mashhad, Iran

${ }^{c}$ Associate professor, Faculty of Economic and Administrative Science, Ferdowsi University of Mashhad, Mashhad, Iran

\section{H R O N I C L E A B S T R A C T}

Article history:

Received May 12, 2013

Received in revised format

12 August 2013

Accepted 28 September 2013

Available online

October 82013

Keywords:

Theorizing

Multi-level models

Levels of analysis

Focal unit

\begin{abstract}
Any phenomenon can be considered and analyzed in terms of different perspectives. In multilevel theorists view, structure or structures of the studied phenomenon are used to consider or to analyze it, completely. Level of analysis indicates the purpose of a researcher or theorist that is intended to be explained or justified, like individual, group or organizational levels and then they are generalized. Contrary to multilevel approach, the conventional approach of theorizing considers micro level or macro level. It cannot perform a simultaneous micro-macro level analysis. A multilevel approach characterized by inter-level and multilevel organizational view to organizational phenomena is an attempt to expand the boundaries of knowledge and provide a new plan. This study uses documentary studies to analyze the multi-level approach of theorizing, multilevel models and multilevel analysis.
\end{abstract}

\section{Introduction}

Much of the research performed in the field of management and organization is associated with problem analysis at one level. For instance, these studies are executed at individual, group, organizational or regional and national levels. However, there are increasing numbers of problems associated with organizations and their management as well as their associated theories, it is necessary and inevitable to address multilevel analysis, and researchers should pay special attention to this problem. Many researchers as of management and organizations recommended that their studied phenomenon had specific complexity that should have been considered in a dynamic system or even with regard to social systems. This causes researchers to pay special attention on analysis at micro, macro or middle levels. However, it is noted that the emphasis on one level and problem analysis from one point of view may cause insufficient or incomplete understanding (Klein \&

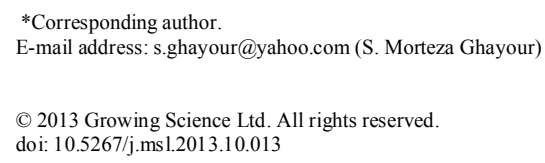


Kozlowski, 2000). Complete implementation of various research techniques by researchers in the field of management and organization is inevitable. If these methods are implemented properly, they may raise several interesting questions (Scott, 1974). Meanwhile management researchers need innovation in research methods to provide new characteristics of management and organization problems. In addition, researchers should look to reconsider basic assumptions. That's why a new method is required to address the research questions. In other words, research methods should become more prominent and closer to real-world problems (Trim \& Lee, 2004).

Organizations are normally multilevel systems and this axiom the foundation of organizational systems theory is reflected in the earliest cases of organizational theory, including the Hawthorne Studies (Roethlisberger \& Dickson, 1939), Homans's theory of groups (1950), Lewin's field theory (1951), sociotechnical systems theory (Emery \& Trist, 1960), Likert's theory of organizational effectiveness (1961), Thompson's (1967) theory of organizational rationality, and Katz and Kahn's (1966) social organizational theory, to name but a few. Further, this axiom continues to provide a foundation for virtually all contemporary theories of organizational behavior. Yet, in spite of the historical tradition and contemporary relevance of organizational systems theory, its effect is merely metaphorical. The system is sliced into organization, group, and individual levels, each level the province of various disciplines, theories, and approaches. The organization may be an integrated system, but organizational science is not (Klein \& Kozlowski, 2000).

One interesting point presented by some researchers is associated with the fact that a research-based management would appear, gradually. According to Gruber and Niles, managing will be increasingly research-based in future. They stated that research-based management was a type of management practice by informed managers. Therefore, new knowledge for management is constantly being developed. Research-based management is associated with a continual process of change in the practice of management (Miner, 2007).

\section{The necessity of theorizing}

It is always an important action to provide an overview of the theorizing concepts before explaining about the multilevel analysis (Bacharach, 1989). Theory provides a framework for the knowledge and having some insights of the important relationships in different scientific fields. Theories developed in the field of management are very essential and in comparison with other social sciences are relatively new. Theories in the field of organization and management offer new and important insights that make a better and fundamental understanding of the management phenomena (Smith \& Hitt, 2005). Dubin (1969) was one of the few researchers who first introduced the relative importance of theorizing in science. He suggested that the primary objective of this theory is on the human mind or the need to theorizing is associated with human need to understand the fact and knowledge. About 60 years ago, Lewin also stated: "there is nothing as practical as a good theory". Van de Ven reiterated this point. In other words, a good theory is practical to the extent that increases knowledge and builds a theory (Trim \& Lee, 2004).

Theories are useful issues to respond to questions and concerns raised by the researchers rather than responding the practical problems. In fact, they are some responses to practical problems, although some theories have special applications that bring about unique status. The advantage of this type of practice-based theory is that it is mostly based on scientific concepts formed by a theoretical logic (Miner, 2007). Theories are provided to give meaning to different phenomena. There are several definitions of theories, for instance Kerlinger (1979) defines theory as: "A coherent set of general propositions used as principles of explanation (control) of the apparent relationships of certain observed phenomena". On the other hand, Weick (1995) defines theory as: "an assessment of a phenomenon related to structures and propositions". He believes that a theory is never perfect and is always on the move in the path of progress and development. 


\section{Types of theory}

Theory has various meanings and definitions and different typologies are presented for theory. According to the theory of analytic processes, theory is classified into four categories including inductive, deductive, model-building and grounded theories. Others classified theories according to the level of inclusion and generalizability and put them in to four categories: Gran Theory, General Theory, Middle-Range Theory and Substantive Theory summarized as follows (de Vijver et al., 2008):

\subsection{Grand Theory}

Grand Theory is a system of interrelated abstract concepts and propositions, which describes a wide range of phenomena. Grand theory concentrates on certain universal laws, which causes theorist to understand the phenomenon of relationships between concepts of phenomena. Parsons's theory of social structure and theories associated with material, time, space, and energy are among some examples of the Grand theories, which are considered in this category. The primary objective of Grand Theory is to form the body of knowledge (Weick, 1999).

\subsection{General Theory}

General theories are like Grand Theories with the difference that these theories more concentrate on a specific phenomenon instead of considering various phenomena. General theories can be described as follows: A general coherent narrative of mental assumptions, arguments and reflections, which describe and define basic structures. This phenomenon deals with the relationship between structure and its boundaries in a broad area used for general phenomenon. Therefore, the theory also focuses on a specific phenomenon and includes all its dimensions (Staw, 1991).

\subsection{Middle-Range Theory}

Middle-Range Theory has a limited scope and range of application can be compared with grand theory and general theory. Middle-Range Theory is formed in the context of a general theory, which is studied in various academic fields. In this type of theory, one concept that is a part of the phenomenon is studied. In other words, the totality of a phenomenon and all its aspects is considered as the subject of general theory while certain concept of phenomenon is considered in the MiddleRange Theory. It provides a detailed explanation of a concept, which could be examined and verified. Boundaries of Middle-Range Theory are clearer and brighter than a general theory. Although boundaries of Middle-Range Theory are included in general theory it becomes too limited in order to focus on a simple concept and its structures. Basic structures of Middle-Range Theory are derived directly from the constituent concepts of general theory and relationships between structures are defined by proposition. Socio-technical theory is an example of Middle-Range Theory, which describes the concept of effective organizations (de Vijver et al., 2008).

\subsection{Substantive Theory}

Substantive theory is based on the Middle-Range Theory, which seeks for accurately describing the relationships among structures of middle-range theory. Substantive theory represents operationalizing (making practical) of general theory. Variables are the basic elements of substantive theory defined as observable entities. Variables may also be considered as an operational combination of one structure (practical form) (Smith \& Hitt, 2005). 


\section{What are multilevel models?}

Organizations by definition have multilevel structures and Individuals work together in teams to form departments, which in turn may be part of a branch or subsidiary of a larger organization, which then is operating within a specific industry in a specific nation. Therefore, theories are needed to explain the behavior of individuals, dyads, groups, departments, organizations, organizational alliances and industries (Dansereau \& Yammarino, 2005).

Addressing the appropriate level of a theory in organizational settings is not merely an academic question, but it has some important practical implications. Because of the pressure for organizational survival and continues growth, a failure to explain, to predict and to influence behavior, appropriately at the various level can be costly and potentially fatal for any organization (Klein et al., 1999). As a consequence of these pressures, organizational researchers have been aware of level issues for some time and the literature discussing the level of theory and analysis issues in the United States and western European organizations are rich and diverse (Yammarino et al., 2008).

Multilevel Models began to be widely applied in the 1980s. The models were first applied to study issues such as school effectiveness in two-level structures of pupils in schools, or to study three-level nested structures, such as pupils in classes in schools, where the response was interval a test score. Multilevel models have also been implemented to investigate variations in response variables for individuals in households, or individuals in areas of varying scales, and they are useful for multivariate and longitudinal studies. Initially, some models for interval responses were developed, and recently developments for generalized linear models have been made, including multilevel logistic models. Multilevel Models are now applied for non-nested situations, including cross classified models, multiple membership models, and multiple membership multiple classification models (Fielding \& Goldstein, 2006). Monte Carlo Markov Chain (MCMC) is often used for these more complex cases. Multilevel models can sometimes be useful for social network analysis: especially for dyadic relationships, ego-networks, and to account for network on a response variable. Whether a multilevel model or another kind of statistical model is appropriate for a particular social network analysis depends on the targets of inference of the analysis, the available data, and the assumptions made about the network structure (Carey \& Wang, 2001).

\subsection{Assumptions of multilevel models}

Fitting a model with untenable assumptions is as senseless as fitting a model to data, which are knowingly flawed (Singer \& Willett, 2003). According to Klein et al., (1994), three different assumptions can be differentiated: homogeneity, heterogeneity and independence. Homogeneity refers to the homogeneity of subunits within higher level units. This implies that constructs differ between units but not within units. When talking about individuals and groups, it is assumed that group members are sufficiently similar with respect to the construct in question that they may be characterized as a whole. Homogeneity, on the other hand, assumes that phenomena are shared and identical within units and differences only happen between different units (Fischer et al., 2005). The second assumption is called heterogeneity, implying the heterogeneity of subunits within higher level units. Alternative names include 'frog-pond', within-group or parts effect (Dansereau \& Yammarino, 1998). Comparative or relative effects, but not absolute effects are important. A frog may be comparatively small in a big pond, but the same frog would appear large when the pond was smaller. Therefore, the assumption is that effects are context-dependent. Any score and its effect will depend on the respective level of scores in the unit of interest (Dansereau et al., 1999). The final assumption is independence, implying that subunits are independent from higher level units. It assumes that individuals are free of group influence. This assumption underlies many statistical tests (e.g., individual scores are independent from each other) and this is the assumption that is more familiar to psychologists. Group membership is irrelevant and the only true variation is between-individual 
variation (individual differences, personality). It is assumed that these constructs can be used to describe individuals and that these constructs represent more or less stable personality traits. Therefore, group membership or social context variables are expected to be of minor importance (Yammarino \& Dansereau, 2008).

\section{Focal unit and level of theory}

Focal unit concept is the central point about level of a theory. Focal units are available entities in which a theory can be generalized such as individuals, groups and organizations. It is relatively easy to distinguish among individuals and populations, but it is too difficult to determine the exact boundaries where a population is finished and another population tarts (such as groups) and the point we cross a level such as a small business unit and arrive another level like organization. It is too difficult to make such a difference among different teams, the communities, networks, strategic alliances, virtual enterprises and multinational companies. These problems along with related challenges provide a definition of focal units of a theory and level of analysis (Rousseau, 1985).

Once a unit was defined as a focal point for generalizing the results, we can build a multilevel theory. This means that predictions can be made about the existence of the central unit and determine how they are related to each other (Chen et al., 2003). Rousseau provided a useful framework to consider the levels of theory, measurement, and analysis for structures, simultaneously. Level of theory refers to the focal level in which generalizations are applied while level of measurement refers to the unit to which the data are directly attached. However, the level of analysis is the unit to which data are assigned for hypothesis testing and statistical analysis (Hitt et al., 2007).

The majority of studies on organizations are conducted by individual analysis as the unit of observation and analysis. The problem is that the unit of analysis should be changed from the individual observation level to the system level. The method of changing these levels is often difficult and uncertain while most organizational studies are not sensitive to this issue. Considering the fact that organizations are independent centers in which individuals interact and compare themselves, in most analyses, an individual is considered as the unit of analysis (Porter, 1996).

Hitt et al. (2007) suggested that every researcher always has faced a decision as to which level of analysis to adopt. Next, they explained that choosing a single level, sometimes could lead to misalignment between the levels of theory and analysis, particularly when predictors distributed at various levels. Fortunately, different methods have been developed for analyzing multilevel data, such as within and between analysis and cross level operator techniques. Although these techniques have bolstered multilevel research throughout the past two decades, recent developments have proven to be more flexible and appropriate for multilevel data arrangements. It seems to be more appropriate to use qualitative methods in these cases (Pfeffer, 1991).

According to Staw (1991), the gap between micro and macro issues has been established and not only researchers classify their groups into micro or macro groups but also even students consider this kind of distinguish in their academic work or plan to choose the data and its interpretation. Unfortunately, this kind of classification has had adverse effects on researches (Guo \& Zhao, 2000).

\section{Basic principles of multilevel theory}

Multilevel Analysis using the hierarchical linear model, random coefficient regression analysis for data with several nested levels and each level is (potentially) a source of unexplained variability. Multilevel analysis is a suitable approach to take into account the social contexts as well as the individual respondents or subjects (Snijders \& Bosker, 2012). Principles of theory building are more seriously considered to build and test the multilevel theory. The power and strength of multilevel theory is in its complexity. These theories pay much attention to organizational realities. Study of 
multilevel phenomenon in organizations is faced complexity of the interactional effect of methods and statistics on one hand and formation or evolution of the theory on the other hand. Multilevel approach for designing and construction of multilevel models and theories follows a few basic principles. These principles in terms of what, how, where, when and why questions are presented in Table 1 as follows (Rezayian \& Ganjali, 2009):

Table 1

Basic principles of multilevel theory

\begin{tabular}{|c|c|c|}
\hline Perspective & Principle & Description of principle \\
\hline \multirow[t]{2}{*}{ What } & First & $\begin{array}{l}\text { Multilevel theorizing must be started with determining the theoretical explanation (definition) of the studied } \\
\text { phenomenon and its creation or production of phenomenon }\end{array}$ \\
\hline & Second & $\begin{array}{l}\text { Multilevel theoretical models can be used for much of organizational phenomena. However, the use of } \\
\text { multilevel models is not necessary in some cases: } \\
1 \text { - When the phenomenon under study is not affected by the organizational unit level. } \\
2 \text { - When the phenomenon under study, performance or behavior does not reflect a lower } \\
\text { level of organizational units. } \\
3 \text { - When the phenomenon under study in the organization literature has been analyzed. }\end{array}$ \\
\hline \multirow[t]{2}{*}{ How } & Third & $\begin{array}{l}\text { Almost all organizational phenomena emerged in a context of higher level or have mostly direct impact on } \\
\text { the process and results of the modulator at the lower level. Related underlying factors and impacts from } \\
\text { higher levels should be considered as part of the theoretical model. }\end{array}$ \\
\hline & Fourth & $\begin{array}{l}\text { Many higher-level phenomena are the result of individual characteristics, cognition, behavior, affect, and } \\
\text { interaction among individuals. Conceptualization of the phenomenon emerged at higher levels and has to } \\
\text { determine theoretically the nature and the processes in bottom-up form. }\end{array}$ \\
\hline \multirow[t]{3}{*}{ Where } & Fifth & $\begin{array}{l}\text { Specifying the nature of the units (formal vs. informal) should be considered in theorizing and explaining } \\
\text { phenomena. Specifying informal entities that are outside the formal or informal boundaries organization or } \\
\text { those happening in formal units that can be separated and special and accurate attention. }\end{array}$ \\
\hline & Sixth & $\begin{array}{l}\text { Considering the intensity and strength of the relationship, and connection between different levels (similar, } \\
\text { adjacent, contained, embedded or mixed together two by two) is necessary. }\end{array}$ \\
\hline & Seventh & $\begin{array}{l}\text { Context (ground) that is considered as basis of the structures on different levels should be a link between the } \\
\text { other contexts. }\end{array}$ \\
\hline \multirow[t]{3}{*}{ When } & Eight & $\begin{array}{l}\text { Time territory will affect many phenomena, whether the top-down and bottom-up process or processes that } \\
\text { will emerge at the same time. A theory should consider the time problem and consider timely providing of } \\
\text { necessary points. }\end{array}$ \\
\hline & Ninth & $\begin{array}{l}\text { The difference in time scale causes top-down processes influence the low-level phenomena faster while } \\
\text { bottom-up processes influence occurs in a longer time period. Research projects must be sensitive to the time } \\
\text { requirements of the theory. }\end{array}$ \\
\hline & Tenth & $\begin{array}{l}\text { Strong communication and relationship between levels can be undermined over time. Theorists should } \\
\text { consider and operate the period of time in the research design. }\end{array}$ \\
\hline Why & Eleventh & $\begin{array}{l}\text { Multilevel theoretical models must explain why questions about itself (and pre-assumptions and basic } \\
\text { assumptions). It should not only explain the 'why' questions but also answers "why not" questions. }\end{array}$ \\
\hline
\end{tabular}

\section{Why multilevel models should be used?}

Multilevel models have various advantages and the most important advantage is that multilevel models provide a suitable framework for the study of multilevel data. The mentioned framework indicates a systematic analysis on how measured independent variables influence various levels of hierarchical structure and it shows interactions among various levels of the dependent variables (Klein \& Kozlowski, 2000). New insights might be obtained by applying micro level theories to phenomena usually examined at the macro level. Some empirical studies are provided to test the conceptual models that have already been developed. Some studies illustrated how multilevel thinking was informing new conceptual developments in the field of management. These studies include micro-level issues of organizations social change (focusing on psychological and social psychological bases), relational and network issues and the macro level involving political, economic, institutional and societal dynamics (de Vijver et al., 2008). However, the effect of micro-level analysis on macro-level and vice versa should be considered.

\subsection{The effect of micro-level analysis on the macro level}

Staw (1991) explained that in the 1960's and 1970' organizational research was considered to be an interdisciplinary fields with theoretical inputs of anthropology including psychology, sociology, political science and economics. With the development of these analyses, this field obtained some achievements and raised and separated as a discussion of specific issues. Psychologists and their 
students trained in related topic have considered issues such as job attitudes, work motivation, absenteeism at work, turnover and stress as pure psychological topics in the micro-level organizational behavior. Sociologists also offered their views on organizational behavior at the macro level in issues such as organizational structure, power, strategies and environment (Khastar, 2010). If organizational actions to be considered only as individual behavior, undoubtedly psychological theories are useful for describing the concepts, especially individuals need some kind of night to deal with external organizations. One of the points in arguments against psychological theories to explain behavior of organizational actors is that behavior of individuals is not independent. Individuals may be socialized to the point that they are acting as full agents of corporations. They serve as the corporate objectives of the organization and their goals should be the organization's goal. Therefore, they are simply looking for benefits for the larger system. Sometimes individual's behavior can be defined as their conformity and loyalty. Staw (1991) offered two discussions on the relationship between the individual and the organization for explanation of micro from micro behavior. The most basic idea is that many micro-level actions are actually behaviors in individual coverage. In this regard, a relatively weak issue is associated with understanding the micro index in organizational actions.

According to existing studies, the study of organizations has most frequently started from the individual as the unit of observation. The problem, however, is that the analysis must move from the individual level of observation to the system level where the problem of interest usually lies. How to do so is often problematic, and much of the study of organizations remains insensitive to this issue. In spite of the fact that we know that organizations are relational entities, in which individuals interact and compare themselves with others, many of our analyses take the individual alone as the unit of analysis and do not attempt to incorporate notions of social structure into either theory or empirical research (Khastar, 2010).

\subsection{The effect of macro-level analysis on micro level analysis}

Pfeffer (1991) discussed structural views on organizational theories and its importance and noted that the least part of the structural views in management studies was the focus on the social structure and its effect on organizations and their individuals. According to Pfeffer (1991), organization theories pay attention to the issues that explain how individuals affect community and community affects individuals. He noted that in sociology, as in economics, assumptions often rest on psychological principles but the distinction is that the prediction and theoretical ideas explain patterns of social relationships in terms of properties of social structure, not in terms of the assumptions made, whether or not these are derivable from psychological principles (Pfeffer, 1991).

\section{Types of multilevel models}

The primary assumption of multilevel modeling is to use a model to describe the data, which means that there was a hierarchical structure in the sampling design. Creation of such structure is conducted by determination of separate models for each level. According to Khastar (2010), there are four categories of models used in multilevel research as follows:

1. Cross-level models: These models describe the relationship between the dependent and independent variables at different levels. Most cross-level model theories of organizations investigate the effects of organizational or group factors on individual behavior or attitude.

2. Mixed effects models: These models suggest that organization interference may influence organization's multiple levels. For instance, the organization changes from a piece of work to a group incentives-based system may change the organization whole image to the outside observer, dynamics of collaborating groups within the organization and job satisfaction of employees . 
3. Mixed determinants models: These models suggest that forecasters at various levels may affect interested criteria. For example, market characteristics (e.g. available job), group characteristics (e.g. variation between groups), and individual characteristics (e.g. job satisfaction) may influence on the transfer of manpower.

4. Multilevel models: Multilevel models explain patterns of recovered relationships between the levels of analysis.

\section{Application Areas of multilevel models}

The notion of individuals, or any other type of objects, that are naturally nested in groups, with membership in the same group leading to a possible correlation between the individuals, turned out to be very compelling in many disciplines, which can be explained by (de Leeuw \& Meijer, 2008):

\subsection{Survey Data}

Many surveys are not based on simple random samples from a relatively homogeneous population, but they are obtained from nested sampling in heterogeneous subgroups. Larger units (e.g., states) are drawn first; within these larger units, smaller units (e.g., counties) are drawn next; and so forth (Satorra \& Muthen, 1995). The reason for such a complicated nesting structure of surveys is to assume that the units were different in some respect. It is then natural to model the heterogeneity between groups through multilevel models (Chambers \& Skinner, 2003).

\subsection{Repeated Measures}

In repeated measures models (including growth study models) we have measurements on a number of individuals that are replicated at a number of fixed time points (longitudinal models). Usually there is only a single outcome variable, but the generalization to multivariate outcomes is an straightforward task (Verbeke \& Molenberghs, 2009).

\subsection{Twin Studies}

In school-based attainment studies, we often deal with a small number of rather large groups. However, the opposite can also occur, either by the nature of the problem or by design. We can decide to use only a small number of students from each class or, in repeated measures studies, we can only have two measurements per individual ("before" and "after", for instance, with a treatment in between) (Maydeu and Böckenholt, 2005).

\subsection{Meta Analysis}

In meta analysis, data, including historical data, are much more accessible than in the past. Many data sets are online or are included in some way or another with published research. This makes it attractive to use previous data sets studying the same scientific problem to get larger sample sizes and perhaps a larger population to generalize. Such (quantitative) analysis of data or results from multiple previous studies composes meta analysis (Raudenbush, 2002).

\subsection{Multivariate Data}

There is a simple way to fit general multivariate data into the multilevel framework. If we have $n$ observations on $m$ variables, one can think of these $m$ observations as nested in $n$ groups with $m$ group members each and then building the model with the usual regression components and a suitable specification for the dispersion of the within group disturbances (Goldstein, 2011). In standard multilevel models, parameters such as regression coefficients are the same for different observations 
within the same group, whereas in multivariate analysis, this is rarely the case. Thus, writing the latter as a multilevel model requires some care (Lehtonen, 2005).

\section{Discussion and conclusions}

Micro level analysis is concentrated on people, their methodologies, dynamic certain relationship and social systems especially on the social issues dealing with face to face interaction examined at the macro level of large systems. In addition to micro and macro level analysis, the middle-range analysis is focused on intermediate or middle level at various areas of sociology and many of them are focused on their own theoretical aspects. There is actually no dependent micro, macro or middle level. Although these analyses can be used, separately, studies on relationship complexity, interactions, social and institutional phenomena indicated that they were not sufficient alone and issues should be examined in terms of different perspectives. Multilevel analysis and the theory provide researchers with a tool to consider various aspects of the problems considering the research focal unit and level of theory. However, the mentioned limitations of analysis method should also be considered.

\section{References}

Bacharach, S. B. (1989). Organizational theories: Some criteria for evaluation. Academy of management review, 14(4), 496-515.

Carey, V. J., \& Wang, Y. G. (2001). Mixed-Effects Models in S and S-Plus. Journal of the American Statistical Association, 96(455), 1135-1136.

Chambers, R. L., \& Skinner, C. J. (Eds.). (2003). Analysis of survey data. John Wiley \& Sons.

Chen, G., Mathieu, J. E., \& Bliese, P. D. (2003). A framework for conducting multi-level construct validation. Research in Multi Level Issues, 3, 273-303.

Dansereau, F. E., \& Yammarino, F. J. (1998). Leadership: The multiple-level approaches: Contemporary and alternative. Elsevier Science/JAI Press.

Dansereau, F., Yammarino, F. J., \& Kohles, J. C. (1999). Multiple levels of analysis from a longitudinal perspective: Some implications for theory building. Academy of Management Review, 24(2), 346-357.

Dansereau, F., and Yammarino, F. J. (Eds.). (2005). Multi-level issues in strategy and methods. Elsevier.

de Leeuw, J., \& Meijer, E. (Eds.). (2008). Handbook of multilevel analysis. Springer.

Fielding, A., \& Goldstein, H. (2006). Cross-classified and multiple membership structures in multilevel models: an introduction and review. University of Birmingham

Fischer, R., Ferreira, M. C., Assmar, E. M. L., Redford, P., \& Harb, C. (2005). Organizational Behaviour across Cultures Theoretical and Methodological Issues for Developing Multi-level Frameworks Involving Culture. International Journal of Cross Cultural Management, 5(1), 27-48.

Goldstein, H. (2011). Multilevel statistical models. Wiley.

Guo, G., \& Zhao, H. (2000). Multilevel modeling for binary data. Annual review of sociology, 441462.

Hitt, M. A., Beamish, P. W., Jackson, S. E., \& Mathieu, J. E. (2007). Building theoretical and empirical bridges across levels: Multilevel research in management. Academy of Management Journal, 50(6), 1385-1399

Kerlinger, F. N. (1979). Behavioral research: A conceptual approach. New York: Holt, Rinehart and Winston.

Khastar, H. (2009). Multilevel approach in organization and management studies. Culture management. No. 19, 160-174

Klein, K. J., Dansereau, F., \& Hall, R. J. (1994). Levels issues in theory development, data collection, and analysis. Academy of Management Review, 19(2), 195-229. 
Klein, K. J., \& Kozlowski, S. W. (2000). From micro to meso: Critical steps in conceptualizing and conducting multilevel research. Organizational research methods, 3(3), 211-236.

Klein, K. J., \& Kozlowski, S. W. (2000). Multilevel theory, research, and methods in organizations: Foundations, extensions, and new directions. Jossey-Bass.

Klein, K. J., Tosi, H., \& Cannella, A. A. (1999). Multilevel theory building: Benefits, barriers, and new developments. Academy of Management review, 24(2), 248-253.

Lehtonen, R. (2005). Multilevel Statistical Models. Journal of the American Statistical Association, 100(469), 353-354.

Maydeu Olivares, A., \& Böckenholt, U. (2005). Structural equation modeling of paired comparison and ranking data. Psychological methods, 10(3), 285.

Miner, J. B. (2007). Organizational behavior. ME Sharpe.

Pfeffer, J. (1991). Organization theory and structural perspectives on management. Journal of Management, 17(4), 789-803.

Porter, L. W. (1996). Forty years of organization studies: Reflections from a micro perspective. Administrative Science Quarterly, 262-269.

Raudenbush, S. W. (2002). Hierarchical linear models: Applications and data analysis methods (Vol. 1). Sage.

Rezaeian, A. And Ganjali, A. (2009). Multilevel approach and its application in theory building of organization. Management Thought. No. 2, 11-12

Rousseau, D. M. (1985). Issues of level in organizational research: Multi-level and cross-level perspectives. Research in organizational behavior, 7(1), 1-37.

Satorra, A., \& Muthen, B. (1995). Complex sample data in structural equation modeling. Sociological methodology, 25, 267-316.

Scott, W. G. (1974). Organization theory: A reassessment. Academy of Management Journal, 17(2), 242-254.

Smith, K. G., \& Hitt, M. A. (Eds.). (2005). Great minds in management: The process of theory development. Oxford University Press.

Staw, B. M. (1991). Dressing up like an organization: When psychological theories can explain organizational action. Journal of Management, 17(4), 805-819.

Tom Snijders, T. And Bosker, R. (2012). Multilevel Analysis: An Introduction to Basic and Applied Multilevel Analysis.2nd edition. Sage.

Trim, P. R., \& Lee, Y. I. (2004). A reflection on theory building and the development of management knowledge. Management Decision, 42(3/4), 473-480.

van de Vijver, F. J., Van Hemert, D. A., \& Poortinga, Y. H. (Eds.). (2008). Multilevel analysis of individuals and cultures. CRC Press.

Verbeke, G., \& Molenberghs, G. (2009). Linear mixed models for longitudinal data. Springer.

Weick, K. E. (1995). What theory is not, theorizing is. Administrative Science Quarterly, 40(3), 385390.

Weick, K. E. (1999). Theory construction as disciplined reflexivity: Tradeoffs in the 90s. Academy of Management Review, 24(4), 797-806.

Yammarino, F. J., Dionne, S. D., Schriesheim, C. A., \& Dansereau, F. (2008). Authentic leadership and positive organizational behavior: A meso, multi-level perspective. The Leadership Quarterly, 19(6), 693-707.

Singer, J. D., \& Willett, J. B. (2003). Applied longitudinal data analysis: Modeling change and event occurrence. Oxford university press.

Yammarino, F. J., \& Dansereau, F. (2008). Multi-level nature of and multi-level approaches to leadership. The Leadership Quarterly, 19(2), 135-141. 